\title{
Crecimiento de una plantación de Nothofagus nervosa durante 20 años en Valdivia
}

\author{
Growth of a plantation of Nothofagus nervosa \\ in Valdivia in a 20-year period
}

\author{
René Reyes Ga, Víctor Gerding ${ }^{\mathrm{b} *}$, Claudio Donoso $\mathbf{Z}^{\mathrm{b}}$ \\ aAgrupación de Ingenieros Forestales por el Bosque Nativo, casilla 1305, Arauco 600B, Valdivia, Chile \\ *Autor de correspondencia: bUniversidad Austral de Chile, casilla 567, Valdivia, Chile, vgerding@uach.cl
}

\begin{abstract}
SUMMARY
The aim of this research was to analyze the growth of a 20-year old plantation of Nothofagus nervosa ex situ (39 $45^{\prime} \mathrm{S}, 7^{\circ} 09^{\prime}$ $07^{\prime \prime} \mathrm{W}, 125 \mathrm{~m}$ asl) and to determine the causes of its spatial variation. In three sites (1: area of highest growth, quadratic mean diameter $=16.5 \mathrm{~cm}$, dominant height $14 \mathrm{~m} ; 3$ : area of lowest growth, quadratic mean diameter $=8.1 \mathrm{~cm}$, dominant height $8 \mathrm{~m}$; and 2: intermediate growth area, quadratic mean diameter $=9.1 \mathrm{~cm}$, dominant height $9 \mathrm{~m}$ ), the plantation was characterized by a) diameter, height, shape, health, vitality, and volume of each tree; b) the diameter growth by using an increment borer; c) content of nutritional elements of foliage; and d) morphological, physical, and chemical characterization of the soil. The estimated volume in site $1\left(112 \mathrm{~m}^{3} / \mathrm{ha}\right.$ ) was eight and four times bigger than that for sites 3 and 2, respectively. Lower growth in sites 2 and 3 is explained by the scarce side protection against radiation supplied by adjoining woods and, in site 3 , it may also be explained by greater resistance of the soil to penetration and less rooting depth. Health and nutritional status of stands did not explain growth differences.
\end{abstract}

Key words: raulí, Nothofagus nervosa, site, growth, soil.

\section{RESUMEN}

Este trabajo tuvo como objetivo analizar el crecimiento de una plantación ex situ (3945’ S, 7309’07” O, 125 m s.n.m.) de Nothofagus nervosa de 20 años y determinar las causas de su variación espacial. En tres sitios (1: área de mayor crecimiento, $\mathrm{DMC}=16,5 \mathrm{~cm}$, altura dominante $14 \mathrm{~m} ; 3$ : área de menor crecimiento, DMC $=8,1 \mathrm{~cm}$, altura dominante $8 \mathrm{~m}$; y 2: área intermedia, $\mathrm{DMC}=9,1 \mathrm{~cm}$, altura dominante $9 \mathrm{~m}$ ) se caracterizó la plantación a través de: a) diámetro, altura, forma, sanidad, vitalidad y volumen de cada árbol; b) el crecimiento diametral mediante tarugos de incremento; c) el contenido de elementos nutritivos del follaje, y d) caracterización morfológica, física y química del suelo. El volumen (ssc) estimado en el sitio 1 (112 $\mathrm{m}^{3} / \mathrm{ha}$ ) fue ocho y cuatro veces mayor que en $\operatorname{los}$ sitios 3 y 2, respectivamente. Los menores crecimientos en los sitios 2 y 3 se explican por la falta de protección lateral otorgada por bosques aledaños contra la radiación y, en el sitio 3, también por la mayor resistencia del suelo a la penetración y la menor profundidad de arraigamiento. La sanidad y el estado nutricional de los rodales no explicaron las diferencias de crecimiento.

Palabras clave: raulí, Nothofagus nervosa, sitio, crecimiento, suelo.

\section{INTRODUCCIÓN}

Las plantaciones de especies nativas, con fines de restauración o productivos, han ido en aumento durante los últimos años debido, entre otras razones, a incentivos económicos especiales por parte del Estado, a una creciente conciencia ambiental y a la apertura de nuevos mercados para las maderas nativas. Una de las especies con mayor valor en estos aspectos es Nothofagus nervosa (Phil.) Dimitri et Milano (raulí). Desde esta perspectiva, es fundamental sistematizar la información disponible sobre el tema en
Chile e ir generando nuevos conocimientos a partir de las plantaciones realizadas durante las últimas décadas.

En Chile, $N$. nervosa crece naturalmente desde el sur de la provincia de Curicó (río Teno, $35^{\circ} \mathrm{S}$ ) hasta el sur de la provincia de Valdivia (40³0'S) (Loewe et al. 1998) por la Cordillera de Los Andes, y en la Cordillera de la Costa desde Cauquenes $\left(35^{\circ} 58^{\prime}\right.$ S) hasta el norte de la provincia de Llanquihue $\left(41^{\circ} \mathrm{S}\right)$ (Sepúlveda y Stoll 2003). En la Cordillera de la Costa la distribución de $N$. nervosa es fuertemente discontinua (Donoso 1978, Grosse y Quiroz 1999). Desde 1952, año en que se registra la primera 
plantación de $N$. nervosa en Chile (fundo Quechumalal, Panguipulli), se han realizado varias experiencias de plantaciones con esta especie en distintas zonas y regiones del país (Espinosa et al. 1977, 1988, Vita 1977, Donoso et al. 1993a, 1999, Meneses et al. 1991, Hernández 1996, Gutiérrez 2004). En general, los crecimientos obtenidos en la precordillera y cordillera andina, dentro del área de distribución natural de la especie, son mayores que los observados en la Depresión Intermedia y Cordillera de la Costa. Un caso particular ocurre con plantaciones realizadas en la Isla de Chiloé, ya que sus crecimientos fueron muy similares a los de la precordillera andina de la provincia de Valdivia. Esto se debería a que en dicha zona el clima presenta una fuerte influencia oceánica y, por lo tanto, el periodo de sequía estival es más breve o inexistente (Donoso 1981), lo cual favorece a la especie si el suelo está bien drenado.

En 1983 se realizó una plantación de $N$. nervosa en la comuna de Valdivia, lugar que está fuera del área de distribución natural de la especie (Donoso 1978, Grosse y Quiroz 1999), con el propósito de estudiar su comportamiento ex situ dada la alta calidad de su madera y el potencial productivo que representa para muchas zonas donde no crece en forma natural. En esta plantación llamaron la atención las grandes diferencias de crecimiento entre distintos sectores del rodal. En este contexto, el objetivo del presente estudio fue realizar un diagnóstico de la plantación para evaluar las diferencias de crecimiento existentes y determinar sus causas, partiendo de la hipótesis que el suelo tiene una influencia importante en ello, ya que el clima y el material genético pueden considerarse constantes en la plantación.

\section{MÉTODOS}

Área de estudio. El clima se caracteriza por precipitaciones que varían entre 2.000 y $2.500 \mathrm{~mm}$ anuales, por lo que existen excedentes de humedad para el consumo de la vegetación, aunque el periodo seco puede durar hasta dos meses (Schlatter et al. 1995). La variación interanual de la precipitación entre los años más lluviosos puede alcanzar valores superiores al 50\% de desviación respecto al promedio normal. La temperatura media anual oscila entre 9 y $12{ }^{\circ} \mathrm{C}$. La amplitud media mensual entre las temperaturas medias extremas fluctúa entre 5 y $8^{\circ} \mathrm{C}$ (IREN 1978). De acuerdo con el clima, el rasgo vegetacional dominante es el de los tipos forestales siempreverde, roble-raulí-coigüe y coigüe-raulí-tepa.

La plantación se encuentra en una loma de un terreno de altitud intermedia, ondulado a quebrado (predio Las Palmas ${ }^{1}, 39^{\circ} 45^{\prime}$ S, $73^{\circ} 09^{\prime}$ O, 125 m s.n.m.). El sitio

1 Propiedad de la Universidad Austral de Chile, ubicada a 18 kilómetros de Valdivia, por la ruta que une dicha ciudad con la de San José de la Mariquina. presentó anteriormente una plantación de Pinus radiata D. Don que fue cosechada en 1982 utilizando tractor articulado, lo que provocó deterioro estructural del suelo (compactación y remoción) en algunos sectores. En 1983 se efectuó la plantación mixta de $N$. nervosa, Laurelia sempervirens (Ruiz et Pavón) Tul. y Persea lingue Nees a un distanciamiento medio de $2 \times 2$ metros, realizándose replantes en 1984 y 1985 . El área ocupa una superficie de 1,5 hectáreas y su exposición general es Sur-Suroeste. Debido a la fuerte mortalidad de $L$. sempervirens y $P$. lingue la plantación quedó compuesta casi en un 100\% por $N$. nervosa a un distanciamiento de 4 x 4 metros (González 1995).

Desde la primera evaluación, en 1984, se observó una gran variación en el crecimiento, vigor y desarrollo de $N$. nervosa en distintos sectores del área plantada. Para la realización de este estudio se delimitaron tres sitios: de mayor crecimiento (sitio 1), de crecimiento intermedio (sitio 2) y de menor crecimiento (sitio 3). Cada sitio fue descrito según sus características topográficas, vegetacionales y edáficas (Schlatter et al. 2003). En todos los sitios el drenaje externo del suelo fue moderado a lento.

El sitio 1 se ubicó en una ladera media, cóncava a recta, con exposición Suroeste y pendiente de $21 \%$. Este sitio se localizó colindante con un rodal de $15 \mathrm{~m}$ de ancho de $P$. radiata de 25 años de edad, con altura de $20-25 \mathrm{~m}$ y DAP de $24-50 \mathrm{~cm}$; adyacente había una plantación de Sequoia sempervirens (D. Don) Endl. de 31 años de edad con altura media de 25 metros y DAP de 37 a $55 \mathrm{~cm}$. Ambas plantaciones de coníferas fueron establecidas 6 y 11 años antes, respectivamente, que la plantación de $N$. nervosa, por lo que fueron consideradas para evaluar la protección lateral desde el noroeste y oeste. El sitio 2 se localizó en la cumbre, en un terreno plano, y el sitio 3 en una ladera alta convexa, con exposición Sur-Suroeste y pendiente de 27\%. En los sitios 2 y 3 no hubo protección lateral.

Caracterización de la plantación. En cada sitio se delimitó una parcela circular de 10 metros de radio $\left(314 \mathrm{~m}^{2}\right)$, donde se caracterizó cada árbol según (julio de 2003): DAP, altura total, forma, sanidad, posición sociológica y vitalidad. El volumen se estimó utilizando la función ajustada por Barría (1996) $\left(\mathrm{R}^{2}=95 \%\right)$ :

$$
\ln (\mathrm{V})=-9,915295+0,937855 * \ln \left(\mathrm{H}^{*} \mathrm{DAP} \mathrm{P}^{2}\right)
$$

donde: $\mathrm{V}=$ volumen del árbol $\left(\mathrm{m}^{3} \mathrm{ssc}\right), \mathrm{H}=$ altura total (m) y DAP $=$ diámetro a $1,3 \mathrm{~m}(\mathrm{~cm})$.

Para el análisis de los crecimientos medio anual y anual periódico se escogieron tres árboles por parcela, dos con DAP similar al diámetro medio cuadrático y otro con DAP máximo; a cada individuo se le extrajeron dos tarugos a 0,3 metros de altura, que representan el crecimiento en los lados norte y sur del fuste, en los cuales fueron medidos los anillos de crecimiento. 
La sanidad del fuste se evaluó como: a) buena: sin daño aparente; b) regular: con síntomas de daños bióticos; c) mala: con signos de daños bióticos en el fuste. La forma de los fustes se caracterizó como: a) buena: fuste recto sin bifurcaciones; b) regular: bifurcado sobre tres metros de altura o torceduras leves (desplazamiento del eje vertical $\leq 1$ radio); c) mala: fuste bifurcado en los primeros tres metros de altura o con fuertes torceduras (desplazamiento del eje vertical $>1$ radio). La vitalidad fue evaluada en las categorías de exploración, degeneración, estancamiento y resignación (Roloff 1993), dependiendo de la estructura y forma de la copa, la cantidad de verticilos y ramillas existentes, y la disposición y abundancia de las hojas en la parte alta de la copa.

En los sitios 2 y 3 fueron seleccionados cinco árboles dominantes en torno al perfil de suelo descrito, a los cuales se les extrajo una muestra de follaje (fines de diciembre de 2002) para su análisis. En el sitio 1 se seleccionaron sólo tres árboles dominantes debido a la dificultad que impuso su mayor altura. El follaje se tomó en el tercio superior de la copa (parte soleada) y se mezclaron las hojas de los árboles de cada sitio. El follaje de los árboles se analizó química y morfológicamente. Se determinaron los contenidos totales de nitrógeno, fósforo, potasio, calcio, magnesio, hierro, manganeso, cobre, zinc y boro (Sadzawka et al. 2001) y mediante una tabla Munsell se caracterizó el color de las hojas.

Para evaluar la influencia de la protección lateral brindada por las plantaciones de $P$. radiata y $S$. sempervirens sobre el desarrollo de $N$. nervosa, se trazaron tres transectos paralelos de 100 metros cada uno, separados por $10 \mathrm{~m}$ entre sí, desde el límite con estas plantaciones hacia el interior de la plantación de $N$. nervosa. Se obtuvieron valores de DAP y altura de acuerdo con la distancia creciente al borde de la plantación protectora. Con tales datos se ajustaron sendas curvas de altura y DAP con respecto a la distancia hacia las plantaciones protectoras.

Caracterización del suelo. En cada sitio se describió un perfil de suelo a través de una calicata (Schlatter et al. 2003). Se determinó la densidad aparente del suelo a distintas profundidades (tercios superior, medio e inferior del perfil) a través del método del cilindro y se midió la resistencia a la penetración, mediante un penetrómetro de punta plana, en cada horizonte (diez repeticiones por estrato).

De cada uno de los primeros cuatro horizontes identificados (aproximadamente 0-50 $\mathrm{cm}$ de profundidad) en los perfiles se obtuvo (Sadzawka et al. 2000): pH (en agua y $\mathrm{KCl} 0,1 \mathrm{~N}$ ), carbono total (oxidación con dicromato de potasio y ácido sulfúrico, determinación colorimétrica), nitrógeno total (digestión Kjeldahl, determinación colorimétrica), fósforo Olsen (disponible); aluminio, calcio, magnesio, sodio, potasio, hierro, cobre, zinc y manganeso (extraíbles en acetato de amonio a pH 4 más DTPA); boro disponible (en extracto $\mathrm{CaCl}_{2}$ 0,15\% a ebullición); aluminio intercambiable (en $\mathrm{KCl} 1 \mathrm{M}$ ); azufre disponible (en fosfato de calcio). En los horizontes inferiores se determinó solamente carbono total y aluminio intercambiable.

En el sitio 1 se presentó el siguiente perfil de suelo con textura franca a franca arcillosa y drenaje interno moderado: A $(0-8 \mathrm{~cm})$, de estructura granular a subpliédrica, friable a firme y color 7,5YR3/2; AB $(8-30 \mathrm{~cm})$ de estructura subpliédrica, friable y color 7,5YR3/1; BA $(30-53 \mathrm{~cm})$, de estructura masiva, friable a muy friable y color 5YR3/3; B1 $(53-84 \mathrm{~cm})$, de estructura masiva, friable a muy friable y color 7,5YR3/3-3/4; B2 (84->120 $\mathrm{cm}$ ), de estructura masiva, friable a muy friable y color 7,5YR4/3-3/3; en este suelo se estimó una capacidad de agua aprovechable de $270 \mathrm{~mm}$ hasta un metro de profundidad. En el sitio 2 el suelo de textura franca a franca arcillosa y drenaje interno moderado era: A (0$19 \mathrm{~cm}$ ), de estructura subpliédrica y granular, friable y color 7,5YR3/2; AB $(19-31 \mathrm{~cm})$, de estructura subpliédrica, friable y color 7,5YR3/4; BA $(31-46 \mathrm{~cm})$, similar al anterior, pero con estructura masiva; B1 $(46-97 \mathrm{~cm})$, de estructura masiva, friable a firme y color 7,5YR3/4; B2 $(97->110 \mathrm{~cm})$, de estructura masiva, friable a firme y color 7,5YR3/4-4/4; se estimó una capacidad de agua aprovechable de $230 \mathrm{~mm}$ hasta un metro de profundidad. El perfil del sitio 3, con suelo franco arcilloso a arcilloso, era: A $(0-12 \mathrm{~cm})$, de estructura subpliédrica y granular, friable y color 7,5YR3/2-3/3; BA (12-24 cm), de estructura subpliédrica, friable y color 7,5YR3/3; B1 $(24-57 \mathrm{~cm})$, de estructura masiva, friable y color 7,5YR4/3; B2 (57$85 \mathrm{~cm}$ ), de estructura masiva, friable y color 7,5YR4/4; 2BC $(85-110 \mathrm{~cm})$, de estructura masiva, friable a firme, de color 7,5YR5/4-6/4, de textura franca arcilla arenosa y con $40-50 \%$ de pedregosidad; $2 C(110->120 \mathrm{~cm})$, de estructura masiva, friable a firme, de color 10YR6/47,5 YR6/4, de textura franca arcilla arenosa y con 1-5\% de pedregosidad; la capacidad de agua aprovechable hasta un metro de profundidad se estimó en $170 \mathrm{~mm}$. El drenaje interno era moderado en superficie variando a moderadolento a partir de los $85 \mathrm{~cm}$ de profundidad.

De acuerdo con CIREN (1999), los suelos pertenecen a la serie Los Ulmos (Typic Palehumult), que corresponden a rojo arcillosos haloisíticos profundos, originados a partir de cenizas volcánicas antiguas depositadas sobre el complejo metamórfico de la costa. Sin embargo, en los sitios 1 y 2 es posible que haya influencia de cenizas volcánicas menos antiguas, como lo descrito para la serie Correltué (Andic Palehumult), la cual se presenta intermitentemente en esta zona sur de su distribución (CIREN 1999). Ello quedaría reflejado en las texturas algo menos arcillosas y colores un poco más claros con respecto al suelo del sitio 3. También la topografía del sitio 3 , una ladera de mayor desgaste natural por su inclinación, exposición y forma, habría impedido la acumulación de cenizas más recientes.

La densidad aparente tendió a ser mayor en el suelo del sitio 3 , pero sin diferencia significativa $(P>0,05)$ con los otros suelos. En cambio, la resistencia a la penetración 


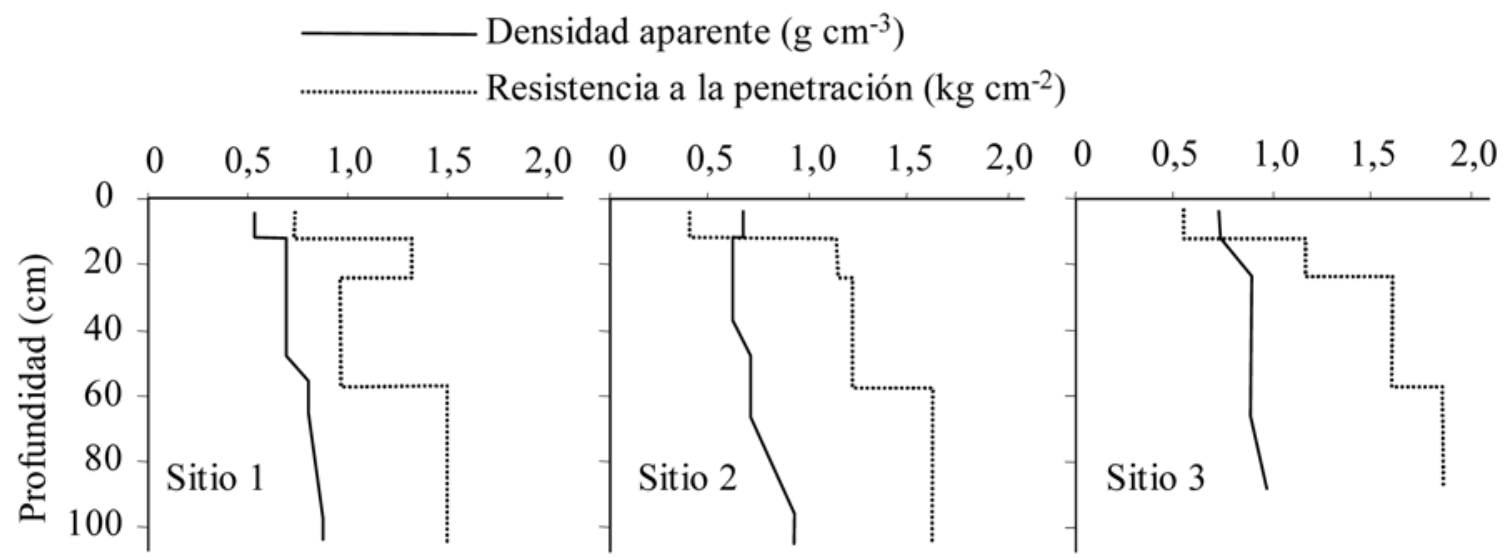

Figura 1. Variación en profundidad de la densidad aparente y la resistencia a la penetración del suelo en cada sitio.

Bulk density and soil resistance to penetration in each site.

mostró valores mayores $(P<0,05)$ en el suelo del sitio 3 , bajo los $24 \mathrm{~cm}$ de profundidad, en comparación con el suelo del sitio 1. En el sitio 2 se encontró una situación intermedia (figura 1). En el caso del sitio 3, a partir de una profundidad de $24 \mathrm{~cm}$ el suelo presentó resistencia a la penetración superior a $1,5 \mathrm{~kg} \mathrm{~cm}^{-2}$, coincidiendo con menor presencia de raíces.

Los suelos eran moderadamente ácidos a fuertemente ácidos, con alto potencial de acidificación, ricos en materia orgánica y nitrógeno total, con adecuada relación $\mathrm{C} / \mathrm{N}$; presentaban suministro medio a bajo de bases y microelementos. La disponibilidad de fósforo y azufre fue baja, especialmente en los sitios 2 y 3 . En estos dos sitios también se observaron bajos niveles de zinc desde el segundo horizonte. En los tres suelos se encontraron altos niveles de aluminio extraíble, que se asocian a la fijación del fósforo. La saturación de aluminio en estos suelos era alta, indicando condiciones desfavorables por toxicidad de aluminio (cuadro 1). En los sitios 2 y 3, con menor crecimiento de $N$. nervosa, el suelo era más pobre en elementos nutritivos, especialmente en fósforo, azufre y zinc.

En los tres sitios se presentó un mantillo de la forma de humus mull (Schlatter et al. 2003), formado por un horizonte Oi, un incipiente Oe y sin Oa. En los sitios 1 y 2 el mantillo cubría toda la superficie del suelo con un espesor de 2 - $3 \mathrm{~cm}$, en cambio en el sitio 3 el recubrimiento era parcial y con menor espesor $(1-2 \mathrm{~cm})$. En todos los casos el material formador correspondía mayoritariamente a hojas de $N$. nervosa, ramillas y restos foliares del sotobosque, con estructura suelta. En el sitio 1 la presencia de raíces finas fue muy abundante en la zona de transición entre Oi y A; en el sitio 2 este tipo de raíces fue común y en el sitio 3 muy escaso (Schlatter et al. 2003). Sólo en el mantillo del sitio 1 se observaron abundantes hifas de hongos.
Análisis estadístico. La relación de la forma, la sanidad y la vitalidad de los árboles de $N$. nervosa con el sitio se analizó con la prueba de independencia Chi cuadrado $(P$ $<0,05)$. Las comparaciones entre medias se realizaron a través de análisis de varianza y la prueba de Tukey $(P<$ $0,05)$. Las relaciones entre variables se evaluaron a través de correlación de Pearson y regresión lineal (Hartung et al. 2002).

\section{RESULTADOS}

Características dasométricas y crecimiento de los rodales. La densidad de los rodales mostró una secuencia decreciente en el orden sitio 2, sitio 1 y sitio 3, resultando el sitio de menor calidad con una densidad del $83 \%$ respecto del mejor sitio (cuadro 2). Como era de esperar, los diámetros y las alturas resultaron directamente proporcionales a la calidad del sitio. Tanto el DAP medio como la altura media de los 300 árboles superiores fueron significativamente mayores $(P$ $<0,05)$ en el sitio 1 que en los sitios 2 y 3 . Estos últimos no se diferenciaron significativamente en estas variables. $\mathrm{El}$ área basal y el volumen en los sitios 2 y 3 resultaron inferiores a los del sitio 1 (cuadro 2), producto de los menores crecimientos y de la menor densidad del rodal en el caso del sitio 3. En el sitio 3 el área basal alcanzó un $20 \%$ y el volumen un $13 \%$ respecto al sitio 1 . Los árboles del sitio 1 crecieron a tasas de $1,04 \mathrm{~cm}$ año ${ }^{-1}$ en diámetro, significativamente más $(P<0,05)$ que los rodales de los sitios 2 y 3 que tuvieron tasas de incremento de 0,59 y $0,50 \mathrm{~cm}^{2} \tilde{n ̃ o}^{-1}$, respectivamente. Entre estos últimos rodales no hubo diferencias significativas $(P>0,05)$ (figura 2$)$. Los crecimientos volumétricos medios fueron de $5,6 \mathrm{~m}^{3}$ $\mathrm{ha}^{-1}$ año $^{-1}$ en el sitio 1 , de 1,4 en el sitio 2 y de 0,7 en el sitio 3. Esto deja en evidencia el escaso crecimiento de los árboles en los sitios 2 y 3. 
Cuadro 1. Características químicas de los suelos en cada sitio.

Chemical characteristics of soils in each site.

\begin{tabular}{|c|c|c|c|c|c|c|c|c|c|c|c|c|c|c|c|c|c|c|c|c|}
\hline \multirow{3}{*}{$\begin{array}{c}\text { Profun- } \\
\text { didad } \\
(\mathrm{cm})\end{array}$} & \multirow{3}{*}{$\begin{array}{c}\mathrm{pH} \\
\text { agua }\end{array}$} & \multirow{3}{*}{$\begin{array}{c}\mathrm{pH} \\
\mathrm{KCl}\end{array}$} & \multirow{3}{*}{$\begin{array}{l}\mathrm{Ct} \\
(\%)\end{array}$} & \multirow{3}{*}{$\begin{array}{l}\mathrm{Nt} \\
(\%)\end{array}$} & \multirow{3}{*}{$\mathrm{C} / \mathrm{N}$} & \multirow{3}{*}{$\begin{array}{c}\mathrm{P} \\
\text { Olsen }\end{array}$} & $\mathrm{Al}$ & $\mathrm{Na}$ & $\mathrm{K}$ & $\mathrm{Ca}$ & $\mathrm{Mg}$ & $\mathrm{Fe}$ & $\mathrm{Mn}$ & $\mathrm{Cu}$ & $\mathrm{Zn}$ & \multirow{3}{*}{ B } & \multirow{3}{*}{$S$} & \multirow{3}{*}{$\begin{array}{c}\mathrm{Al} \\
\mathrm{KCl} \\
\end{array}$} & \multirow{3}{*}{$\begin{array}{c}\text { Suma de } \\
\text { bases } \\
(\mathrm{cmol}+ \\
\left.\mathrm{kg}^{-1}\right)\end{array}$} & \multirow{3}{*}{$\begin{array}{c}\text { Satura- } \\
\text { ción A } \\
(\%)\end{array}$} \\
\hline & & & & & & & \multicolumn{9}{|c|}{ Acetato de Amonio pH 4,8 - DTPA } & & & & & \\
\hline & & & & & & & \multicolumn{9}{|c|}{$\left(\mathrm{mg} \mathrm{kg}^{-1}\right)$} & & & & & \\
\hline \multicolumn{21}{|l|}{ Sitio 1} \\
\hline $0-8$ & 5,60 & 4,71 & 12,0 & 0,53 & 22,8 & 5,0 & 1.351 & 48 & 267 & 398 & 336 & 188 & 72 & 5,0 & 4,4 & 1,1 & 17 & 36 & 5,6 & 6,6 \\
\hline $8-30$ & 5,34 & 4,48 & 10,0 & 0,45 & 22,4 & 3,2 & 2.151 & 42 & 155 & 362 & 112 & 206 & 31 & 4,9 & 0,9 & 1,1 & 3 & 72 & 3,3 & 19,6 \\
\hline $30-53$ & 5,61 & 5,05 & 3,5 & 0,22 & 15,8 & 1,2 & 1.398 & 30 & 71 & 438 & 29 & 109 & 7 & 1,8 & 0,1 & 0,4 & 56 & 17 & 2,7 & 6,4 \\
\hline $53-63$ & 5,52 & - & 6,7 & - & - & - & - & - & - & - & - & - & - & - & - & - & - & 20 & - & - \\
\hline $63-84$ & 5,45 & - & 2,6 & - & - & - & - & - & - & - & - & - & - & - & - & - & - & 27 & - & - \\
\hline $84-120$ & 5,49 & - & 1,6 & - & - & - & - & - & - & - & - & - & - & - & - & - & - & 19 & - & - \\
\hline \multicolumn{21}{|l|}{ Sitio 2} \\
\hline $0-9$ & 5,42 & 4,45 & 11,1 & 0,48 & 23,1 & 3,2 & 1.335 & 35 & 219 & 435 & 245 & 175 & 61 & 4,0 & 1,8 & 1,0 & 2 & 79 & 4,9 & 15,2 \\
\hline 9-19 & 5,32 & 4,32 & 6,6 & 0,31 & 21,2 & 1,2 & 1.657 & 38 & 95 & 406 & 106 & 152 & 21 & 4,2 & 0,1 & 1,2 & 2 & 90 & 3,3 & 23,2 \\
\hline $31-46$ & 5,07 & 4,15 & 2,2 & 0,14 & 15,9 & 0,4 & 1.249 & 36 & 81 & 131 & 55 & 148 & 6 & 4,0 & 0,1 & 0,8 & 32 & 105 & 1,5 & 44,3 \\
\hline 46-97 & 5,10 & - & 1,5 & - & - & - & - & - & - & - & - & - & - & - & - & - & - & 128 & - & - \\
\hline $97-120$ & 4,95 & - & 1,3 & - & - & - & - & - & - & - & - & - & - & - & - & - & - & 159 & - & - \\
\hline \multicolumn{21}{|l|}{ Sitio 3} \\
\hline $0-12$ & 5,30 & 4,53 & 9,3 & 0,40 & 23,3 & 1,8 & 1.742 & 43 & 197 & 672 & 129 & 228 & 36 & 4,6 & 0,7 & 0,8 & 3 & 85 & 5,1 & 15,6 \\
\hline $12-24$ & 5,47 & 4,73 & 6,8 & 0,31 & 21,9 & 1,0 & 1.716 & 37 & 84 & 247 & 67 & 181 & 15 & 4,2 & $<0,1$ & 0,6 & 3 & 18 & 2,2 & 8,3 \\
\hline $24-43$ & 5,84 & 5,21 & 2,9 & 0,14 & 19,9 & 0,2 & 1.119 & 44 & 43 & 517 & 90 & 212 & 6 & 4,2 & $<0,1$ & 0,4 & 33 & 0 & 3,6 & $<0,1$ \\
\hline $43-57$ & 5,88 & 5,30 & 1,7 & 0,09 & 19,2 & 0,1 & 907 & 52 & 26 & 760 & 62 & 189 & 10 & 3,7 & $<0,1$ & 0,4 & 30 & 1 & 4,6 & 0,3 \\
\hline $57-85$ & 5,69 & - & 1,5 & - & - & - & - & - & - & - & - & - & - & - & - & - & - & 2 & - & - \\
\hline $85-110$ & 5,29 & - & 0,9 & - & - & - & - & - & - & - & - & - & - & - & - & - & - & 79 & - & - \\
\hline $110-120$ & 4,88 & - & 1,2 & - & - & - & - & - & - & - & - & - & - & - & - & - & - & 660 & - & - \\
\hline
\end{tabular}

- : No analizado.

Cuadro 2. Características dasométricas en cada sitio.

Dasometric characteristics in each site.

\begin{tabular}{|c|c|c|c|c|c|c|c|c|}
\hline \multirow{2}{*}{ Sitio } & \multicolumn{6}{|c|}{ Todo el rodal } & \multicolumn{2}{|c|}{$\begin{array}{c}\text { Promedio de } 300 \text { árboles } \\
\text { mayores } \mathrm{ha}^{-1}\end{array}$} \\
\hline & Árboles ha-1 & $\begin{array}{l}\mathrm{DMC} \\
(\mathrm{cm})\end{array}$ & $\begin{array}{l}\text { DAP máximo } \\
\quad(\mathrm{cm})\end{array}$ & $\begin{array}{l}\text { DAP mínimo } \\
(\mathrm{cm})\end{array}$ & $\begin{array}{l}\text { Área basal } \\
\left(\mathrm{m}^{2} \mathrm{ha}^{-1}\right)\end{array}$ & $\begin{array}{l}\text { Volumen } \\
\left(\mathrm{m}^{3} \mathrm{ha}^{-1}\right)\end{array}$ & $\begin{array}{l}\text { DAP } \\
(\mathrm{cm})\end{array}$ & $\begin{array}{l}\text { Altura } \\
(\mathrm{m})\end{array}$ \\
\hline 1 & 1.115 & $16,5 \mathrm{a}$ & 29,5 & 4,3 & 23,8 & 112 & $24,4 \mathrm{a}$ & $14 \mathrm{a}$ \\
\hline 2 & 1.273 & $9,1 \mathrm{~b}$ & 18,6 & 2,6 & 8,3 & 28 & $14,1 \mathrm{~b}$ & $9 \mathrm{~b}$ \\
\hline 3 & 924 & $8,1 \mathrm{~b}$ & 16,0 & 2,0 & 4,8 & 14 & $12,0 \mathrm{~b}$ & $8 \mathrm{~b}$ \\
\hline
\end{tabular}

Las letras iguales agrupan sitios sin diferencias significativas entre ellos $(P<0,05)$. En el caso del DMC, la diferencia entre rodales se analizó con respecto al DAP promedio.

En relación al incremento anual en diámetro se observaron tres etapas con tendencias distintas (figura 2). Entre los años 1 y 3 se alcanzaron los mayores incrementos medios en el sitio $1(1,22 \mathrm{~cm}$ año-1), seguido por los sitios 3 y 2 ( 1,0 y $0,78 \mathrm{~cm}_{\text {año }}{ }^{-1}$, respectivamente). Entre los años 3 y 10 las tasas de crecimiento fueron similares en los sitios 2 y 3 , mientras que en el sitio 1 el crecimiento fue mayor. Entre los años 10 y 15 hubo un aumento del crecimiento en el sitio 2 que permitió, en definitiva, que este rodal se encontrara más desarrollado que el del sitio 3 al momento de realizar este estudio.

$\mathrm{El}$ arraigamiento disminuyó en profundidad e intensidad desde el sitio 1 al sitio 3 (figura 3). En este último, el arraigamiento estaba limitado hasta los $40 \mathrm{~cm}$ de profundidad y más abajo la presencia de raíces era escasa. En los suelos de los sitios 1 y 2 el arraigamiento era abundante con una amplia distribución de raíces, ocupando casi la totalidad del perfil. En el sitio 2 el arraigamiento intensivo se observó en el estrato $0-25 \mathrm{~cm}$, mientras que en el sitio 1 se extendió hasta los $45 \mathrm{~cm}$ de profundidad.

Forma, sanidad y vitalidad de los árboles. Los principales defectos de forma correspondieron a bifurcaciones del fuste (cuadro 3). Tanto la forma como sanidad de los árboles fueron independientes del sitio $(P>0,05)$. Sin embargo, las tendencias mostraron que el rodal del sitio 1 presentó 

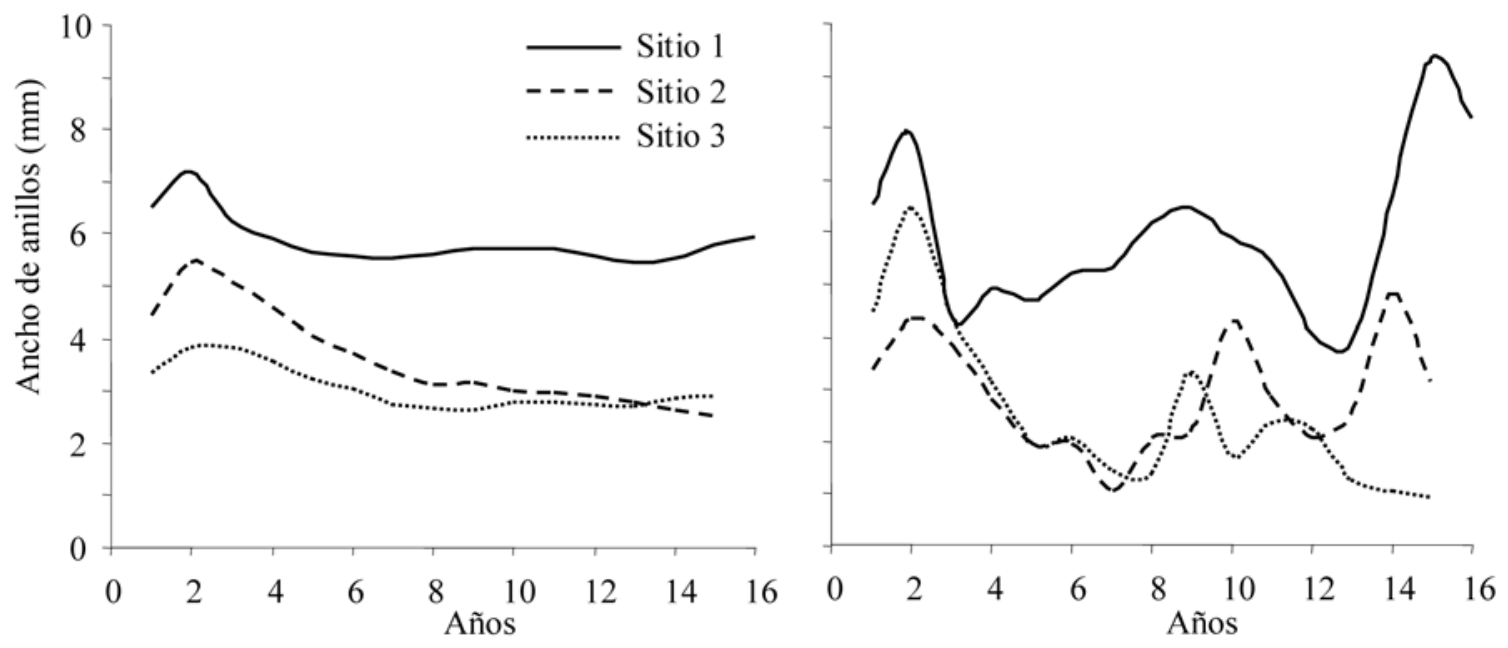

Figura 2. Crecimiento medio anual (izquierda) y corriente anual (derecha) de los árboles en los tres sitios. Las curvas representan valores promedio de tres árboles en cada sitio (faltan los últimos 4 a 5 años debido a problemas en el procesamiento de las muestras).

Mean annual growth (left) and current annual (right) of trees in the three sites. Curves represent mean values of three trees in each site (the last 4 to 5 years are missing due to problems with sample processing).

Densidad de raíces finas (número $\mathrm{dm}^{-2}$ )
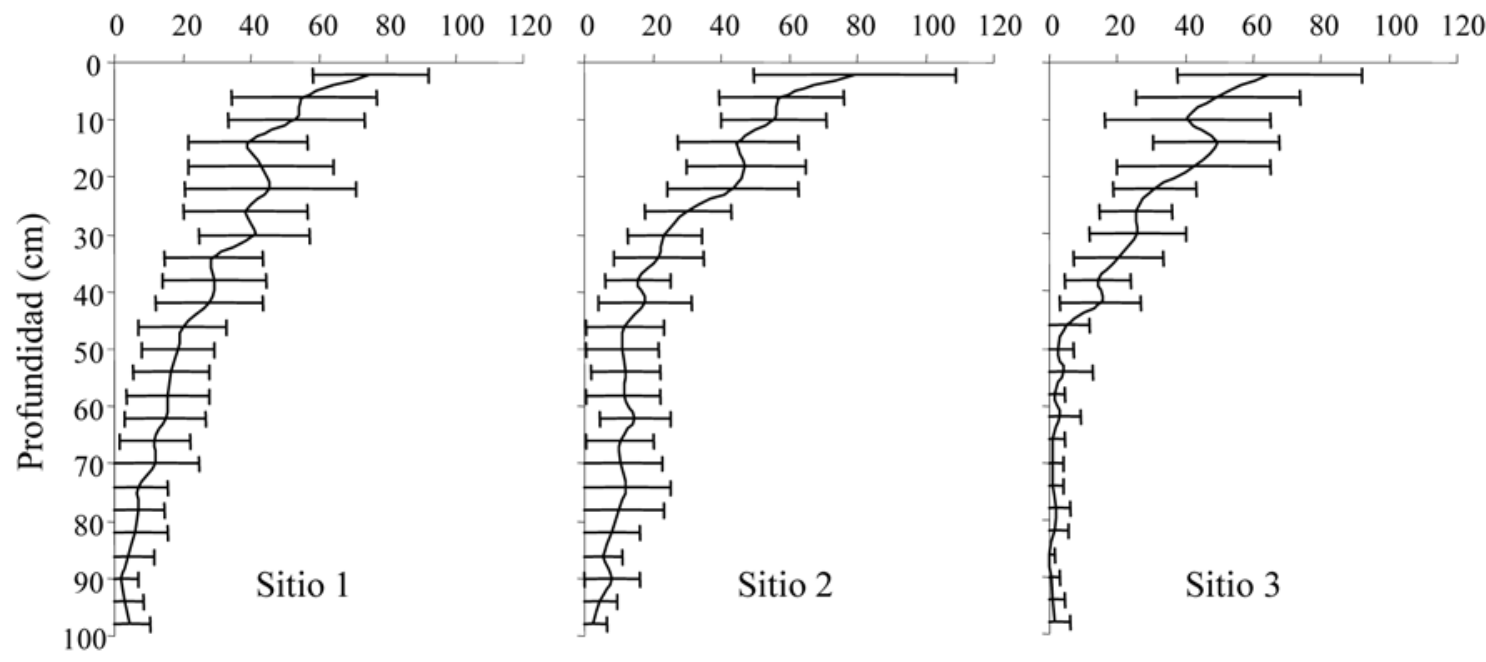

Figura 3. Distribución de raíces finas en el perfil de suelo en cada sitio (media $\pm S$ ).

Distribution of fine roots system in the soil profile in each site (mean $\pm S$ ).

mejor estado sanitario, con más del $70 \%$ de los individuos en buen estado. En cambio, el sitio 3 presentó mejores condiciones de forma con más del $90 \%$ de los individuos en las clases buena y regular. La vitalidad de los árboles (cuadro 3, figura 4) mostró dependencia directa de la calidad de sitio $(P<0,01)$. Todos los individuos del sitio 1 se encontraron en la clase de vitalidad de exploración, lo cual indica que eran árboles vigorosos, frondosos y de buen crecimiento. En los sitios 2 y 3, en cambio, gran parte de los individuos se encontró en las clases de vitalidad de degeneración y estancamiento. El sitio 3 mostró las peores condiciones de vitalidad de los árboles, incluyendo un $8 \%$ de los individuos en la categoría de resignación.
El color del follaje de los árboles del sitio 1 fue más oscuro, dentro del matiz verde (5GY 4/4-3/4 en el haz y 5GY 5/4 en el envés), que en el sitio 2 (5GY 4/6 y 4/8 en el haz y 5GY 5/6-6/6 en el envés), lo que coincide con una mayor vitalidad. En el sitio 3 los árboles presentaban un matiz verde amarillento típico de árboles con menor vitalidad (5GY 5/6-5/8 y 2.5GY 5/6-6/6 en el haz y 5GY 6/4-6/6 y 2.5GY 5/6-6/6 en el envés). Tanto en los aspectos sanitarios como en la vitalidad se apreció un progresivo deterioro desde el sitio 1 al 3 .

En los tres sitios $N$. nervosa presentó buen nivel nutricional con altos contenidos en todos los elementos; una excepción se observó en el sitio 2 con un contenido 
marginal de fósforo (cuadro 4). Los muy altos niveles de manganeso foliar, especialmente en el sitio 3, indican condiciones de alta humedad en el suelo. En el sitio 1 el muy alto contenido de nitrógeno es causante de la mayor relación N/P.

Cuadro 3. Frecuencia (\%) de árboles según forma, sanidad y vitalidad en cada sitio.

Frequency $(\%)$ of trees according to shape, health and vitality in each site.

\begin{tabular}{|c|c|c|c|c|c|c|c|c|c|c|}
\hline \multirow{2}{*}{ Sitio - } & \multicolumn{3}{|c|}{ Forma } & \multicolumn{3}{|c|}{ Sanidad } & \multicolumn{4}{|c|}{ Vitalidad* } \\
\hline & Buena & Regular & Mala & Buena & Regular & Mala & $\mathrm{e}$ & d & $\mathrm{s}$ & $\mathrm{r}$ \\
\hline 1 & 34 & 46 & 20 & 71 & 17 & 11 & 100 & 0 & 0 & 0 \\
\hline 2 & 32 & 27 & 40 & 55 & 40 & 5 & 8 & 79 & 13 & 0 \\
\hline 3 & 48 & 45 & 7 & 52 & 38 & 10 & 0 & 75 & 17 & 8 \\
\hline
\end{tabular}

* (e) exploración; (d) degeneración; (s) estancamiento; (r) resignación (Roloff 1993).
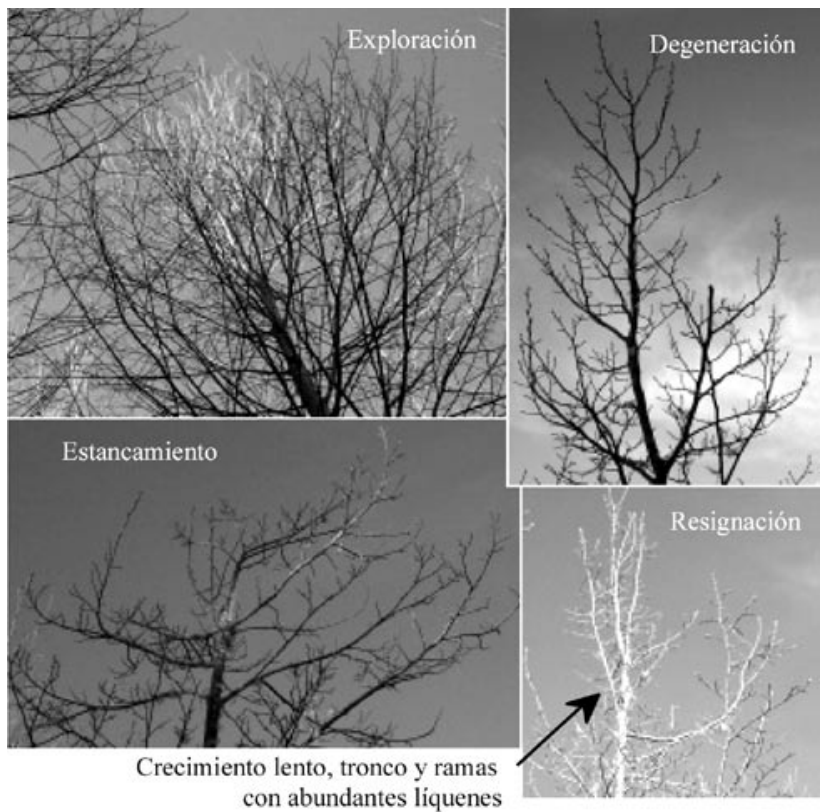

Figura 4. Ejemplos de copas en los distintos grados de vitalidad (según Roloff 1993).

Examples of crowns at different degrees of vitality (according to Roloff, 1993).

Cuadro 4. Contenidos de elementos nutritivos en el follaje. Content of nutritional elements in the foliage.

\begin{tabular}{|c|c|c|c|c|c|c|c|c|c|c|c|}
\hline \multirow{2}{*}{ Sitio } & \multicolumn{6}{|c|}{ Elementos mayores $(\%)$} & \multicolumn{5}{|c|}{ Elementos menores $\left(\mathrm{mg} \mathrm{kg}^{-1}\right)$} \\
\hline & $\mathrm{N}$ & $\mathrm{P}$ & $\mathrm{N} / \mathrm{P}$ & $\mathrm{K}$ & $\mathrm{Ca}$ & $\mathrm{Mg}$ & $\mathrm{Fe}$ & $\mathrm{Mn}$ & $\mathrm{Cu}$ & $\mathrm{Zn}$ & B \\
\hline 1 & 2,46 & 0,15 & 16,4 & 0,73 & 1,00 & 0,21 & 46 & 684 & 7,2 & 40 & 25 \\
\hline 2 & 1,77 & 0,12 & 14,8 & 0,67 & 0,78 & 0,23 & 39 & 534 & 6,2 & 30 & 22 \\
\hline 3 & 1,59 & 0,14 & 11,4 & 0,64 & 0,98 & 0,25 & 39 & 806 & 6,0 & 28 & 20 \\
\hline
\end{tabular}

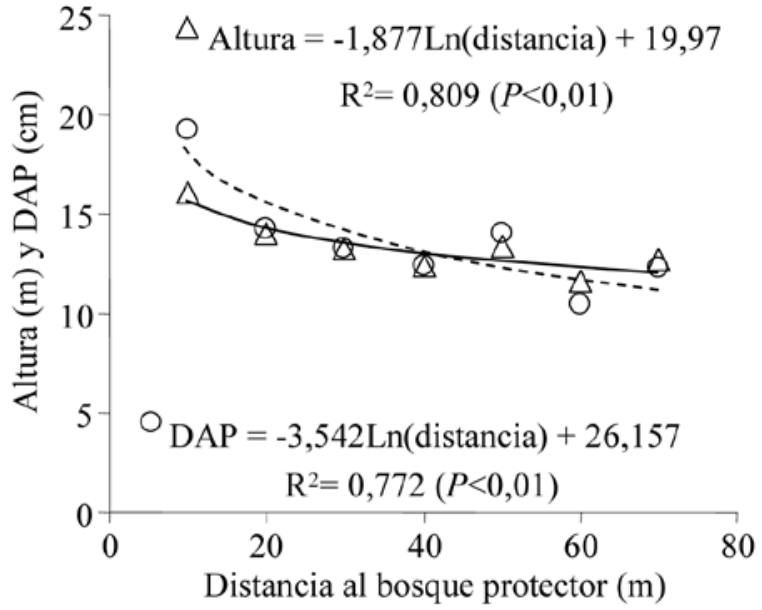

Figura 5. Variación de la altura y del DAP de $N$. nervosa en función de la distancia hacia plantaciones protectoras.

BHD and height variation of $N$. nervosa depending on the distance to the protecting plantations.

Protección lateral. Las plantaciones de $P$. radiata y $S$. sempervirens adyacentes al sitio 1 habrían tenido un efecto de protección lateral para $N$. nervosa (figura 5). Se apreció una disminución paulatina de la altura y del DAP de $N$. nervosa con el aumento de la lejanía a las plantaciones protectoras, especialmente en los primeros $40 \mathrm{~m}$ de distancia.

La altura media varió desde $18 \mathrm{~m}$, junto a las plantaciones protectoras, hasta $13 \mathrm{~m}$ a una distancia de 50 metros de ellas. Sin embargo, el efecto de la protección lateral fue mayor en los primeros $20 \mathrm{~m}$ de distancia (figura 5). El 56\% de la disminución observada en la altura se produjo en los primeros 20 metros desde las plantaciones protectoras y el $80 \%$ se alcanzó a $50 \mathrm{~m}$ de distancia. Para distancias mayores no se encontró una relación significativa $(P>0,05)$ con el crecimiento de $N$. nervosa.

\section{DISCUSIÓN}

En los tres sitios analizados la plantación de $N$. nervosa presentó bajo crecimiento, incluso en el sitio 1, comparado con lo observado en plantaciones de la Cordillera de Los Andes dentro de la distribución natural de la especie (Espinosa et al. 1988, Donoso et al. 1993b, Barría 1996, Hernández 1996). Respecto a plantaciones ex situ en el sur de Chile, esta plantación alcanzó crecimientos superiores en el sitio 1 y similares o menores en los sitios 2 y 3 (Meneses et al. 1991, Gutiérrez 2004).

En los sitios 1 y 2 el suelo presenta condiciones de fertilidad superiores que en el sitio 3. Ello se ve reflejado, principalmente, en el mayor espacio arraigable y mejores regímenes de agua (mayor capacidad de agua aprovechable), de aire (drenaje interno moderado) y de elementos 
nutritivos. El desarrollo radicular, el crecimiento aéreo y la vitalidad de los árboles se vieron afectados en el sitio 3 , donde el suelo presenta menor profundidad fisiológica, mayor resistencia a la penetración, condiciones de aireación restringida y características químicas más limitantes. En comparación, los suelos donde se desarrolla naturalmente N. nervosa (Donoso 1981, Rodríguez et al. 1983, Gerding 2000) son, en general, de texturas medias, consistencia baja, drenaje interno moderado a rápido, sin problemas de aireación y de baja resistencia a la penetración. Es decir, esta especie crece en condiciones estructurales de suelo más favorables que las encontradas en esta plantación, aunque los suelos de los sitios 1 y 2 serían más parecidos a los suelos de origen.

Los suelos en la plantación son más ácidos y de menores contenidos de materia orgánica, pero con mayor disponibilidad de elementos nutritivos, especialmente en los sitios 1 y 2 , con respecto a los suelos de procedencia de las plantas utilizadas (Malleco) (Gerding 2000). Sin embargo, es posible que tales diferencias no sean significativas con suelos de la zona sur de su distribución, donde las condiciones climáticas son similares o más lluviosas que en la zona de la plantación. En todo caso, el suelo del sitio 3 presentó características químicas más restrictivas que en los otros sitios. Los sitios 1 y 2 presentaron suelos de similares características de fertilidad, por lo que se habría esperado que la plantación fuera homogénea en estos dos sitios.

Las diferencias de crecimiento observadas entre los sitios se explican, por una parte, por la fertilidad de los suelos y, por otra, por la protección lateral que ha ejercido el bosque adyacente contra la radiación solar. Esto último explica las diferencias observadas en el crecimiento de la plantación entre los sitios 1 y 2 , ya que en el sitio 1 hubo protección lateral y el sitio 2 no la tuvo. En el sitio 3 tampoco hubo protección lateral y, además, el suelo es más limitante, lo que contribuyó al menor crecimiento de $N$. nervosa. Las diferencias de crecimiento también tuvieron su expresión a través del grado de vitalidad de los árboles definido por Roloff (1993), lo cual permitió caracterizar adecuadamente las distintas situaciones encontradas en los tres sitios.

Durante los tres a cuatro primeros años, después del establecimiento de la plantación, los individuos del sitio 3 crecieron a una tasa anual corriente mayor que en el sitio 2 , lo cual se pudo deber a la exposición Sur-Suroeste del sitio 3, más favorable para el crecimiento de $N$. nervosa por su menor radiación solar y mayor humedad. Junto a ello, en los primeros años aún no se expresaban en el crecimiento las limitaciones del suelo del sitio 3 . A partir del año 12, aproximadamente, las tasas de crecimiento en el sitio 3 disminuyeron fuertemente, en comparación con lo ocurrido en los sitios 1 y 2 , donde se produjeron aumentos. Tal disminución puede explicarse por las limitaciones edáficas de alta resistencia a la penetración y del drenaje interno restringido en el subsuelo, lo que provoca un menor espacio arraigable. Adicionalmente, pudo haber mayor efecto de la vegetación competidora en los árboles menos desarrollados. En cambio, los árboles de los sitios 1 y 2 crecieron en un suelo que les permitió expandir mejor su sistema radicular, alcanzando mayor suministro de agua, incrementando así su tasa de crecimiento. En el sitio 1 la protección lateral y la exposición más sombría habrían favorecido un mejor balance hídrico en comparación con el sitio 2, más expuesto al sol, lo que puede explicar diferencias en las tasas de crecimiento entre estos sitios.

Se aprecia que el efecto de la protección lateral es relevante para el crecimiento de $N$. nervosa en esta área, lo cual fue detectado por Torres (1996) en plantaciones recién establecidas en la zona de Valdivia. La mayor proporción de las diferencias de crecimiento observadas entre los sitios se relacionaría con la protección lateral, más que con variaciones edáficas. Esto es consistente con la autoecología de $N$. nervosa que, a diferencia de otros Nothofagus spp. nativos, es semitolerante y menos agresiva como colonizadora, requiriendo de cierto nivel de protección para desarrollarse adecuadamente (Donoso et al. 1999). En este sentido, el balance hídrico de la plantación sería la principal causa de las variaciones de crecimiento observadas (Gerding y Schlatter 1995, Thiers 2004).

Las condiciones nutricionales de $N$. nervosa en los tres sitios no explican las variaciones de crecimiento observadas. Sin embargo, el menor contenido de nitrógeno en el follaje del rodal del sitio 3, coincidente con hojas de tonalidad verde amarillento, podría ser considerado como una de las causas del menor crecimiento. No obstante, los niveles foliares de elementos nutritivos en los tres sitios serían adecuados para un normal desarrollo de Nothofagus spp. Comparativamente, $N$. nervosa sería más exigente que $N$. dombeyi, según los resultados de Jara (1986), pero menos que $N$. obliqua, el cual alcanza niveles foliares mayores en una amplia zona de su distribución geográfica en Chile (Thiers 2004).

Para una adecuada expansión ex situ de plantaciones de $N$. nervosa la selección del sitio y el manejo silvicultural resultan particularmente importantes. Aunque un sitio sea similar al de algunas áreas donde se desarrollan naturalmente renovales de esta especie (Donoso et al. 1993a), la protección de sombra parcial en los períodos de establecimiento y juvenil puede ser decisiva en el crecimiento del rodal.

\section{CONCLUSIONES}

Nothofagus nervosa mostró un bajo crecimiento en los tres sitios, pero significativamente menor en ausencia del efecto de protección lateral causada por bosques aledaños. También habría mermado el crecimiento un suelo de moderada profundidad fisiológica, con deficiente estructura y limitaciones de aireación. El grado de vitalidad de los árboles se relacionó directamente con la calidad de 
sitio, demostrando que la especie es sensible a pequeñas variaciones de sitio en este ambiente ex situ. La sanidad, la forma y el estado nutritivo de los árboles no se vieron influidos por la calidad del sitio.

\section{AGRADECIMIENTOS}

Este estudio se realizó con el apoyo del proyecto "Ecología y Silvicultura de los Tipos Forestales de la Décima Región" del Convenio CONAF/UACH 1983-2004. Agradecemos a Felipe Leiva, director ejecutivo del CEFOR, por su apoyo en el trabajo de terreno. Por su colaboración en la captación de datos agradecemos al ingeniero forestal Carlos Zamorano, a los estudiantes de ingeniería forestal de la Universidad Católica del Maule, Iván Medel y Lisandro Rocco, y a los estudiantes de ingeniería forestal de la Universidad Austral de Chile, Rodrigo Bravo y Montse Aguilera; a Emilio Cuq y Alexia Wolodarsky, por el procesamiento de los tarugos, y al Laboratorio de Nutrición y Suelos Forestales de la Universidad Austral de Chile, por su aporte en el procesamiento de las muestras de suelo y follaje.

\section{REFERENCIAS}

Barría L. 1996. Comparación de la estructura y crecimiento de una plantación de Roble-Raulí, con una de Roble-RaulíUlmo, en la provincia de Valdivia. Tesis Ingeniería Forestal. Valdivia, Chile. Universidad Austral de Chile, Facultad de Ciencias Forestales. 84 p.

Donoso C. 1978. La silvicultura de Nothofagus en Chile. Berkeley, Estados Unidos. Departamento de Silvicultura y Conservación, Universidad de California. 102 p.

Donoso C. 1981. Tipos forestales de los bosques nativos de Chile. Santiago, Chile. CONAF, FAO, FO:DP/CHI/76/003. Documento de Trabajo $\mathrm{N}^{\circ} 38$. Corporación Nacional Forestal. $83 \mathrm{p}$.

Donoso P, C Donoso, V Sandoval. 1993a. Proposición de zonas de crecimiento para renovales de roble (Nothofagus obliqua) y raulí (Nothofagus alpina) en su rango de distribución natural. Bosque 14(2): 37-55.

Donoso P, T Monfil, L Otero, L Barrales. 1993b. Estudio de crecimiento de plantaciones y renovales manejados de especies nativas en el área andina de las provincias de Cautín y Valdivia. Ciencia e Investigación Forestal 7(2): 255-287.

Donoso P, M González, B Escobar, I Basso, L Otero. 1999. Viverización y plantación de Raulí, Roble y Coigüe en Chile. In Donoso C, A Lara (eds.) Silvicultura de los Bosques Nativos de Chile. Santiago, Chile. Editorial Universitaria, p. 177-244.

CIREN (Centro de Información de Recursos Naturales, CL). 1999. Descripciones de suelos. Materiales y símbolos. Estudio agrológico de la provincia de Valdivia, X Región, Chile. Santiago, Chile. 199 p.

Espinosa M, G Rodríguez, G Medina. 1977. Tres años de crecimiento de una plantación de raulí (Nothofagus alpina) de 25 años de edad. Concepción, Chile. Boletín de Investigación CCF 3, Universidad de Concepción. 19 p.

Espinosa M, J García, E Peña. 1988. Evaluación del crecimiento de una plantación de Raulí (Nothofagus alpina (Poepp. et Endl.) Oerst.) a los 34 años de edad. Agrociencia 4(1): 67-74.

Gerding, V. 2000. Descripción de suelos en la Reserva Nacional Malleco. Informe del proyecto CONAF-ONF. Valdivia, Chile. Universidad Austral de Chile, Facultad de Ciencias Forestales. 23 p.

Gerding V, JE Schlatter. 1995. Variables y factores del sitio de importancia para la productividad de Pinus radiata $\mathrm{D}$. Don en Chile. Bosque 16 (2): 39-56.

González M. 1995. Predio Las Palmas, plantación raulí (Nothofagus nervosa) en 1983. Informe técnico proyecto "Semillas y técnicas de vivero y plantación CONAF-UACH". Valdivia, Chile. Facultad de Ciencias Forestales, Universidad Austral de Chile. 16 p.

Grosse H, I Quiroz. 1999. Silvicultura de los bosques de segundo crecimiento de Roble, Raulí y Coigüe en la región centro sur de Chile. In Donoso C, A Lara (eds.) Silvicultura de los Bosques Nativos de Chile. Santiago, Chile. Editorial Universitaria. p. 95-128.

Gutiérrez NA. 2004. Evaluación del crecimiento y rendimiento volumétricos en ensayos de plantación de Nothofagus obliqua y Nothofagus alpina al aplicar intervenciones silvícolas. Tesis Ingeniero Forestal. Santiago, Chile. Universidad de Chile. 49 p.

Hartung J, B Elpelt, KH Klösener. 2002. Statistik. Lehr- und Handbuch der angewandten Statistik. 13 ${ }^{\mathrm{a}}$ ed. Munchen, Alemania. Oldenbourg Verlag. 975 p.

Hernández E. 1996. Análisis del crecimiento de una plantación de Raulí (Nothofagus alpina (Poepp. et Endl.) Oerst.) en la Precordillera andina de la provincia de Valdivia. Tesis Ingeniero Forestal. Valdivia, Chile. Facultad de Ciencias Forestales, Universidad Austral de Chile. 74 p.

IREN (Instituto de Investigación de Recursos Naturales, CL). 1978. Estudio de suelos de la Provincia de Valdivia. Valdivia, Chile. IREN-Universidad Austral de Chile. 178 p.

Jara GM. 1986. Importancia de los elementos nutritivos Nitrógeno, Fósforo y Potasio en el crecimiento de Coigüe (Nothofagus dombeyi (Mirb.) Oerst) durante sus dos primeros años. Tesis Ingeniero Forestal. Valdivia, Chile. Facultad de Ciencias Forestales, Universidad Austral de Chile. 80 p.

Loewe V, M Toral, G Freitte, M Camelio, M Mery, C López, E Urquieta. 1998. Monografía de Raulí: Nothofagus alpina. Santiago, Chile. CONAF/INFOR/FIA. 90 p.

Meneses M, G Paredes, P Núñez. 1991. Opciones silviculturales para el manejo y utilización del bosque Siempreverde, Décima Región desde Río Bueno al sur. Informe de Convenio $\mathrm{N}^{\mathrm{o}}$ 184. Informe Final. Valdivia, Chile. Facultad de Ciencias Forestales, Universidad Austral de Chile. 103 p.

Rodríguez R, O Matthei, M Quezada. 1983. Flora arbórea de Chile. Concepción, Chile. Ediciones de la Universidad de Concepción. 404 p.

Roloff A. 1993. Kronenentwicklung und Vitalitätsbeurteilung ausgewählter Baumarten der gemässigten Breiten. Band 93. Frankfurt am Main, Alemania. Sauerländer's Verlag. $258 \mathrm{p}$.

Sadzawka A, R Grez, M Mora, N Saavedra, M Carrasco, C Rojas. 2000. Métodos de análisis recomendados para los suelos 
chilenos. Santiago, Chile. Comisión de Normalización y Acreditación, Sociedad Chilena de la Ciencia del Suelo. $32 \mathrm{p}$.

Sadzawka A, R Grez, M Mora, N Saavedra, M Carrasco. 2001. Métodos de análisis de tejidos vegetales. Santiago, Chile. Comisión de Normalización y Acreditación, Sociedad Chilena de la Ciencia del Suelo. 35 p.

Schlatter J, V Gerding, H Huber. 1995. Sistema de ordenamiento de la tierra. Herramienta para la planificación forestal. Aplicado a la X Región. Valdivia, Chile. Serie Técnica, Facultad de Ciencias Forestales, Universidad Austral de Chile. 93 p.

Schlatter J, R Grez, V Gerding. 2003. Manual para el reconocimiento de suelos. Valdivia, Chile. $3^{\mathrm{a}}$ ed. Universidad Austral de Chile. 114 p.

Sepúlveda CA, A Stoll. 2003. Presencia de Nothofagus alpina (Poepp. et Endl.) Oerst. (Fagaceae) en el borde costero de la Región del Maule, Chile Central. Gayana Bot. 60(2): 132-133.

Thiers O. 2004. Roble (Nothofagus obliqua [Mirb.] Oerst.)Sekundärwälder in Zentral- und Südchile: Bestimmung der für die Bestandesproduktivität wichtigen Stamdortfaktoren. Freiburg, Alemania. Freiburger Bodenkundliche Abhandlungen. Heft 42. 170 p.

Torres A. 1996. Grados de cobertura y fertilización en el establecimiento de plantaciones de roble (Nothofagus obliqua (Mirb.) B1.), raulí (Nothofagus alpina (Poepp. et Endl.) Oerst.) y coigüe (Nothofagus dombeyi (Mirb.) Oerst.). Tesis Ingeniero Forestal. Valdivia, Chile. Facultad de Ciencias Forestales, Universidad Austral de Chile. 64 p.

Vita A. 1977. Crecimiento de algunas especies forestales en el Arboretum del Centro Experimental Forestal Frutillar. X Región. Santiago, Chile. Boletín Técnico No 47. Facultad de Ciencias Forestales, Universidad de Chile. 16 p.

Recibido: 21.09 .04

Aceptado: 07.05.07 